\title{
Cue-dependent effects in recall of categorized lists
}

\author{
STEPHEN MADIGAN* \\ University of Southern California, Los Angeles, California 90007
}

\begin{abstract}
Noncued and cued recall of categorized lists are similar in a number of ways: They show the same rate of gain in item recall with increasing category size; they both produce serial position effects within categories; and they both show a "some-or-none" pattern of with-category recall frequency. Cued and noncued recall differ in other respects: There is a great improvement in item and in category recall attendant on the provision of cues, and only noncued recall displays a list position effect. The points of similarity are taken to indicate that both cued and noncued recall measure the same underlying processes. The points of differences suggest the utility of cueing procedures in identifying the origin of characteristics of recall, according to the principle that any feature of noncued recall that is altered by provision of cues must originate in the retrieval process; features resistant to such modification represent encoding or storage effects.
\end{abstract}

This paper describes a simple experimental comparison of cued and noncued recall of categorized word lists. There were two main objects of the study. The first was to assess some similarities and differences in recall performance under cued and noncued conditions. While there are well known and reliable effects of providing category names as cues for recall of category instances (Tulving \& Pearlstone, 1966), the utility of this kind of "horizontal" cueing procedure has been called into question lately by Underwood: “. . . we are not learning about retrieval processes in free recall by the horizontal cueing approach. The procedure circumvents entirely the natural production or retrieval phase [1972, pp. 10-11]." The extent to which cueing techniques such as Tulving and Pearlstone's produce a departure from "normal" free recall can be assessed by examination of the generality of features of recall across cued and noncued conditions, and the present study was designed to allow such comparisons.

A second point of the study was to explore the possible use of cued vs noncued recall comparisons in isolating the locus of certain recall phenomenon. Tulving (1969) reports one such use: The serial position effect of noncued (free) recall can be greatly reduced by the use of cues, and it can be argued that this feature of recall cannot be due entirely to storage factors; instead, it must at least in part depend on conditions of retrieval. In the present study, this kind of proposition was studied with the following general theoretical theme: Any characteristic of noncued recall that is eliminated or altered by provision of cues must be based on the retrieval conditions of noncued recall; effects resistant to such modification may be ascribed to encoding or storage processes.

\section{METHOD}

Design

The experiment was based on a variant of the categorized

*This research was supported by Grant GB-36172 from the National Science Foundation. list-category cued procedure (Tulving \& Pearlstone, 1966). Its novel feature was the use of lists containing categories of varying size or items per category (IPC). Cohen (1966) seems to have been one of the few investigators to use this kind of within-list procedure. Category size (from one to five items per category) and retrieval condition (cued and noncued recall) were the two main variables. The list consisted of 60 to-be-remembered words from 20 different categories. There were four blocks of items, each block containing one category of each of the five category sizes, so that the list contained four replications of the five levels of category size. Seven different versions of the basic list were used. In each of these, categories were assigned randomly to category size conditions. There was further random selection of items within categories and allocation of category size to within-block positions, as well as random ordering of items in a given category.

\section{Procedure}

The 20 categories were chosen from the Battig and Montague (1969) norms. Five items were selected from each category, generally from the 10 most frequent entries. Seven replications of the basic list were videorecorded. Since the category name was presented prior to (blocked) presentation of category items, the actual lists presented consisted of 80 presentations (the 20 category names plus the actual category instances). Both names and items were displayed for 3 sec each.

The study conditions were identical for the cued (CR) and noncued recall (NCR) groups, Ss being told only that there would be a memory test for category items (but not category names) following list presentation. The list was presented on a television monitor. The usual free recall instructions were given to noncued recall $\mathrm{Ss}$; cued recall $\mathrm{Ss}$ received a list of the 20 category names prior to recall. Five minutes of written recall were allowed for each group. Thirty-eight Ss were tested in each condition (CR or NCR), All seven replications of material were used in both treatments.

\section{RESULTS}

\section{Item and Category Recall}

Figure 1 summarizes the effects of cueing condition and category size on item and category recall. Following Tulving and Pearlstone (1966) and Cohen (1966), a category was counted as recalled if at least one item from that category was recalled. There are large and obvious differences between $\mathrm{CR}$ and NCR in both measures. Item recall and category recall are linearly 


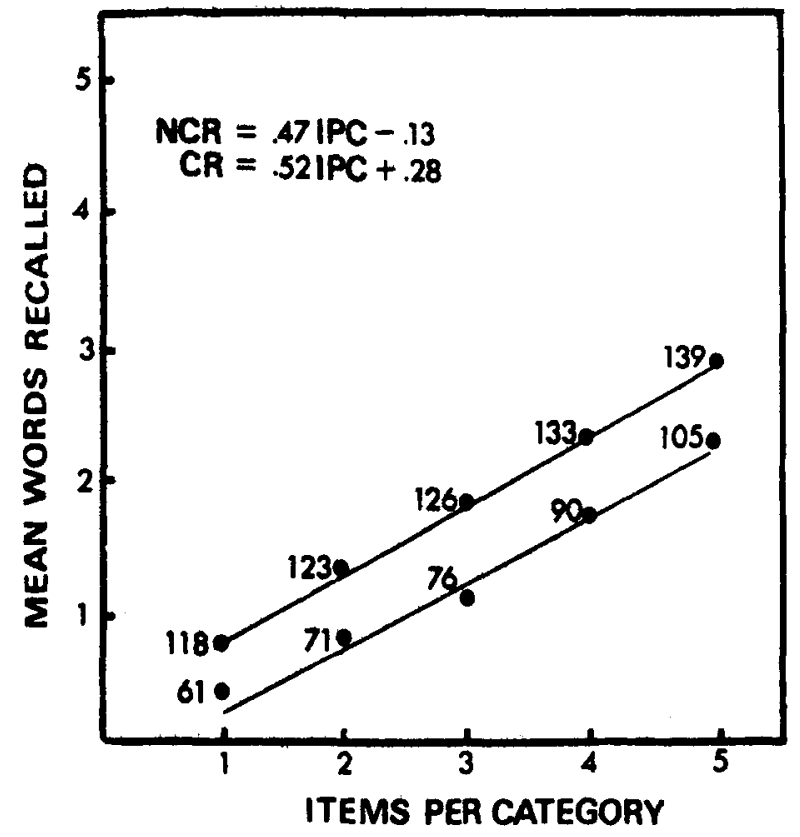

Fig. 1. Mean item as a function of category size in cued and noncued recall. Numbers beside each data point are total category recall frequencies. Standard deviations ranged from .05 (one IPC) to .84 (five IPCs) in NCR and CR conditions.

increasing functions of category size, and cued recall greatly exceeds noncued recall. For item recall, main effects of category size and cue condition (CR, NCR) were significant $(p<.001)$; there was no interaction effect. There were the same main effects for category recall, in addition to an interaction $(\mathrm{p}<.01)$ that was probably only a ceiling effect in CR conditions.

All four sets of data (item recall and category recall under NCR and CR) are easily described as linear functions of category size. The slope estimates for item recall (Fig. 1) in NCR and $\mathrm{CR}$ are very similar $[t(6)=.24, p>.10]$. Slope and intercept estimates for category recall were .07 and .32 for NCR and .03 and .74 for CR. Coefficients of determination $\left(\mathrm{r}^{2}\right)$ were .98 or greater in all cases.

In the cued recall test that followed NCR, Ss nearly doubled the number of items recalled; recall as a function of IPC had the same slope as the CR data, with a slightly greater intercept (.6). The main significance of these data is the large increase in number of new categories added following NCR (a mean of 7.5 per S); they are otherwise nearly identical to the $\mathrm{CR}$ results.

Because of the possibility of extensive generation of list items by Ss in this kind of experiment, it is of some importance to examine the occurrence of intrusions in recall. Table 1 shows the mean numbers of categorical intrusions (items that could be unequivocally classified as belonging to a list category but not presented in the list). Not shown are the unclassifiable intrusions; these means were .16 and .11 for cued and noncued recall groups. The categorical intrusion data are consistent with those reported by Tulving and Pearlstone (1966) in suggesting relatively more intrusions in cued than in noncued recall. They are not consistent with those results in that there was no indication that the number of intrusions in cued recall increased with increasing category size. For present purposes, the main feature of the intrusion data is that the absolute size of the intrusion component in recall is small, with about one-half of all Ss making no intrusions at all. These data do not suggest that the superiority in recall of list words in CR conditions can be attributed to any significant degree to a pure "generate and guess" strategy.

\section{Input Position Effects}

There were serial position effects at the level of blocks within the list. Table 2 shows mean item and category recall scores as functions of list position, collapsed over category size. Standard deviations for item recall were roughly uniform between cueing conditions and across blocks; they varied from 2.43 to 3.34 . For category recall scores, standard deviations ranged from .68 to 1.23. In noncued recall of items and of categories, there were both "primacy" and "recency" effects for the four list segments. Single-factor (repeated-measures) analyses of variance of NCR scores produced $F$ ratios of 8.55 and 7.80 for item and category recall $(\mathrm{p}<.001)$. In contrast, there were no large or reliable position effects for cued recall for either measure $(\mathrm{p}>.05)$.

Table 2 also shows mean item recall per category recalled (Tulving and Pearlstone's $\mathrm{Rw} / \mathrm{c}$ measure-item recall, given category recall). There are two interesting aspects of these data. First, there are no position effects in either cued or noncued recall for this measure. Second, mean recall per category recall was greater in noncued recall than in cued recall. Collapsed across blocks, the differences between means was small (2.39 for NCR, 2.16 for $\mathrm{CR}$ ) but reliable $[\mathrm{t}(74)=2.79$, $p<.01]$.

There was a second kind of position effect as well:

Table 1

Mean Numbers of Categorical Intrusions

\begin{tabular}{lccccc}
\hline & \multicolumn{5}{c}{ Items } \\
\cline { 2 - 6 } & 1 & 2 & 3 & 4 & 5 \\
\hline Noncued Recall & .03 & .26 & .31 & .18 & .11 \\
Cued Recall & .45 & .34 & .31 & .34 & .31 \\
\hline
\end{tabular}

Table 2

Mean Number of Items and Categories Recalled as a Function of Input Block

\begin{tabular}{llcccc}
\hline & & \multicolumn{4}{c}{ Input } \\
\cline { 3 - 6 } & & 1 & 2 & 3 & 4 \\
\hline \multirow{3}{*}{ Items } & NCR & 6.32 & 5.66 & 5.34 & 8.11 \\
& CR & 9.05 & 9.34 & 8.68 & 9.71 \\
Categories & NCR & 2.66 & 2.34 & 2.29 & 3.32 \\
& CR & 4.24 & 4.16 & 4.08 & 4.37 \\
Items Per & NCR & 2.38 & 2.42 & 2.33 & 2.44 \\
Category & CR & 2.13 & 2.25 & 2.13 & 2.22 \\
\hline
\end{tabular}


Recall of the first presented category items was greater than recall of subsequently presented items in the category. Here, however, the position effect was obtained in cued as well as noncued recall. Collapsed over IPC levels from two to five, the proportions of first and last category instances recalled were .48 and .37 in NCR; CR proportions were .72 and .54 .

\section{DISCUSSION}

With respect to the first-stated purpose of this study, the results suggest that there are a sufficient number of similarities between cued and noncued recall to conclude that they do not reflect fundamentally different processes; it may well be possible to understand the normal free recall process with the aid of such procedures. Cued and noncued recall show the same rate of gain with increasing category size, and they both produce serial position effects within categories. They are similar in one other respect that will be briefly mentioned here. Cohen (1966) described noncued recall as having a "some-or-none" characteristic: Either a relatively high proportion of category instances are recalled or none are recalled for a given category. In the present results this was true of both cued and noncued recall. For no category size in either CR or NCR was there anything remotely like a rectangular distribution of number of items recalled; in most instances there was a greater frequency of recall of exactly zero items than recall of a small number of instances.

The main point of contrast between CR and NCR that emerges from these results is, of course, the large difference in overall recall levels. In addition to this effect, there was at least one feature of recall that occurred in noncued recall but not in cued recall (or in noncued recall scores conditionalized on category recall), namely, the list position (input block) effect. Given current interpretations of cueing effects-the alteration of conditions of accessibility of material in memory-the conclusion follows that the position effect in noncued recall reflects the influence of the conditions of retrieval in that experimental condition; it does not arise from features of the storage process. [These data also provide an interesting parallel to Tulving and Psotka's (1971) demonstration that position (RI) effects at the level of whole lists are eliminated by category cueing]. The serial position effect within categories, by the same reasoning, is due to initial encoding or storage processes, since this effect occurred in roughly the same form in both cued and noncued recall. These kinds of outcomes argue for the analytical utility of cued recall procedures in the development of an understanding of "normal" free recall. It is true that the use of categorized lists and category name cueing produce a departure from the free recall test in its "canonical" form; and it also is the case, as Underwood (1972) states, that one has now to account for unaided recall of category names in the same way that one has to account for recall of single items; but it does not seem entirely accurate to characterize cueing studies of this kind as contributing nothing to understanding of the free recall process.

Finally, this study produced one difference between cued and noncued recall that contrasts sharply with previous reports. Tulving and Pearlstone (1966) found that mean item recall, conditionalized on category recall, was identical in both CR and NCR. From this, they arrived at the general theoretical conclusion that the only function of category names was to provide access to larger memory units; they did not affect within-category recall. In the present results, noncued recall was superior to cued recall in terms of this measure (Table 2). While there is no direct way of accounting for this difference, the following is a possible explanation of it: In cued recall tests, the $E$ provided the $S$ with a list of "copies" of the category names originally presented in the input sentence. However, as other kinds of research have lately indicated (Tulving \& Thompson, 1973), these "copies" do not guarantee access to their matches in memory, and it may be that they are less effective than cues successfully generated from memory by Ss in NCR conditions.

\section{REFERENCES}

Battig, W. F., \& Montague, W. E. Category norms for verbal items in 56 categories: A replication and extension of the Connecticut category norms. Journal of Experimental Psychology Monograph, 1969,80.

Cohen, B. H. Some-or-none characteristics of coding behavior. Journal of Verbal Learning \& Verbal Behavior, 1966, 5, 182-187.

Tulving, E. Forgetting: Another look at an old problem. Paper presented at the XX International Congress of Psychology, London, 1969.

Tulving, E., \& Pearlstone, Z, Availability versus accessibility of information in memory for words. $J$ ournal of Verbal Learning \& Verbal Behavior, 1966, 5, 381-391.

Tulving, E., \& Psotka, J. Retroactive inhibition in free recall: Inaccessibility of information available in the memory store. Journal of Experimental Psychology, 1971, 87, 1-8.

Tulving, E., \& Thompson, D. Encoding specificity and retrieval processes in episodic memory. Psychological Review, 1973 , 80, 352-373.

Underwood, B. J. Are we overloading memory? In A. W. Melton and E. Martin (Eds.), Coding processes in human memory. Washington, D.C: Winston, 1972

(Received for publication February 4, 1974; revision received A pril 15, 1974.) 